\title{
Robust Adaptive Tracking Control of the Underwater Robot with Input Nonlinearity Using Neural Networks
}

\author{
Mou Chen ${ }^{1}{ }^{*}$ Bin Jiang ${ }^{1}$, Jie Zou $^{2}$, Xing Feng ${ }^{2}$ \\ ${ }^{1}$ College of Automation Engineering, Nanjing University of Aeronautics and Astronautics, \\ $29^{\#}$ Yu Dao Street, \\ Nanjing, 210016, China \\ E-mail: chenmou@nuaa.edu.cn, bingjiang@nuaa.edu.cn \\ ${ }^{2}$ Key Laboratory of National Defense Science and Technology on Fire Control Technology \\ $25^{\#}$ Kaixuan West Road, \\ Luoyang, 471009, China \\ E-mail:zoujie@163.com,fengxing@163.com
}

Received: $19-10-2009$
Accepted: $31-05-2010$

\begin{abstract}
In this paper, robust adaptive tracking control is proposed for the underwater robot in the presence of parametric uncertainties and unknown external disturbances. Backstepping control of the system dynamics is introduced to develop full state feedback tracking control. Using parameter adaptation, backstepping control and variable structure based techniques, the robust adaptive tracking control is presented for underwater robots to handle the uncertainties, saturation and dead-zone characteristics of actuators. Actuator nonlinearities comprising of dead-zone and saturation are explicitly considered in the tracking control design. Under the proposed tracking control, semi-global uniform boundedness of the closed-loop signals is guaranteed via Lyapunov analysis. Numerical simulation results are given to illustrate the effectiveness of the proposed robust adaptive tracking control.
\end{abstract}

Keywords: Underwater robots, saturation, dead-zone, tracking control, backstepping control

\section{Introduction}

In the marine engineering, various underwater robots and vehicles have been designed and developed for practical applications such as the exploration of deep sea environments, structure and subsurface monitoring, subsea maintenance, and biological surveys. The robust adaptive control for underwater robots with parametric uncertainties and unknown external disturbances is important for achieving the above mentioned tasks. A large number of effective control techniques have been proposed for underwater robots such as robust adaptive control [1], sliding mode control [2], and neural network control [3]. In [1], robust feedback control was proposed for underwater robotic vehicles. Neural network basedtime optimal sliding mode control was presented for an autonomous underwater robot [2]. In [3], robust neural control was studied for underwater robot manipulators. In practice, the system uncertainty

*Corresponding author. Tel: (+86+25) 84893084; E-mail: chenmou@ nuaa.edu.cn. 
and the external disturbance of the underwater robot should be explicitly considered in control design to enhance the disturbance rejection ability of the closed-loop system.

Since marine control applications are characterized by widely changing environmental disturbances and various sea conditions, the dynamics of the underwater robot should be treated as an uncertain multi-input and multi-output (MIMO) nonlinear system. To tackle the unknown term of uncertain nonlinear systems, universal function approximators including neural networks (NNs) have been extensively used in the control design due to their universal approximation capabilities. In [4], an approximation based method has yielded promising results in handling model uncertainties and unknown disturbances for fully actuated ocean surface vessels. Neural network systems for online control adaptation were investigated for the underwater robot [5]. However, the input nonlinearity of the actuator has not been explicitly considered in the control design of the underwater robot.

Actuator saturation and dead-zone are the most important non-smooth nonlinearities which can severely degrade the closed-loop system performance if ignored in the control design. Dead-zone is a static input-output relationship which provides zero output for a range of input values. To handle the actuator dead-zone problem, robust adaptive controls have been proposed for uncertain plants in [6-9]. Adaptive neural control of MIMO nonlinear time varying delay systems with unknown deadzone and gain signs has been developed via the proposed description of a general nonlinear dead-zone model [10]. Adaptive dynamic surface control was proposed to handle a class of pure-feedback nonlinear systems with unknown dead zone and perturbed uncertainties [11]. Similarly, actuator saturation can severely degrade the closed-loop system performance. The analysis and design of control systems with actuator saturation nonlinearities have been studied in [12-14]. A nonlinear control method was proposed to achieve the attitude maneuver of a three-axis stabilized flexible spacecraft with control input nonlinearity [15]. Combined dead-zone and saturation is seldom considered, especially for un- certain nonlinear systems. In this paper, the backstepping tracking control is proposed for the uncertain MIMO nonlinear dynamics of an underwater robot with input dead-zone and saturation.

In recent twenty years, backstepping control has become one of the most popular control techniques for some special classes of uncertain nonlinear systems $[16,17]$. On the other hand, neural network $(\mathrm{NN})$ or fuzzy logical system (FLS) as the approximator has been adopted in the robust control design for the uncertain nonlinear systems [18-26]. Combining backstepping approach with NN to control nonlinear system has received increasing attention [18-25]. In [18], adaptive NN control was investigated for strict-feedback nonlinear systems using backstepping design. Adaptive NN control was developed for nonlinear systems by state and output feedback [19]. In [20], the stable adaptive control was proposed for a class of nonlinear multivariable systems using nonlinearly parametrized function approximators. By exploiting the special properties of affine terms, adaptive neural control for two classes of uncertain MIMO nonlinear systems was proposed [21]. Adaptive backstepping NN control was proposed for a class of affine nonlinear systems in [23]. In [24], stable neural control was developed for unknown nonlinear systems using backstepping control method.

In the tracking control of underwater robots, actuators such as thrusters and rudders which inherently possesses nonlinearities are used. Thus, this work is motivated by the tracking control of the underwater robot with uncertainties, input dead-zone and saturation. The control objective is to track a desired trajectory in the presence of parametric uncertainties and the time varying disturbances. The main contribution of the paper are as follows:

(i) a tracking control in combination with variable structure control (VSC), approximation-based and parameter adaptation techniques is developed which can reduce the requirement on the knowledge of the underwater robot parameters.

(ii) Actuator nonlinearities such as saturation and dead zone are explicitly considered in the control design stage.

(iii) rigorous stability analysis is guaranteed using 
Lyapunov analysis which shows the semiglobal uniform boundedness of all closed-loop signals.

The organization of the paper is as follows. Section 2 details the problem formulation for the tracking control of underwater robots. Section 3 presents the proposed robust adaptive tracking control design considering the dead-zone and saturation nonlinearity of actuators. Simulation studies of the proposed control are shown in Section 4 to demonstrate the effectiveness of our approach, followed by some concluding remarks in Section 5.

\section{Problem Formulation}

Considering the dead-zone and saturation nonlinearity of actuators, the dynamic behavior of an underwater robot with external disturbance and input nonlinearity can be described in the form of [27]

$$
\begin{aligned}
\dot{\eta} & =J(\eta) v \\
M \dot{v}+C(v) v+D(v) v+g(\eta) & =\tau+d(v, \eta, t) \\
\tau & =\Phi(u) \\
y & =\eta
\end{aligned}
$$

where $J(\eta)=\left[\begin{array}{ccc}\cos \left(\eta_{\psi}\right) & -\sin \left(\eta_{\psi}\right) & 0 \\ \sin \left(\eta_{\psi}\right) & \cos \left(\eta_{\psi}\right) & 0 \\ 0 & 0 & 1\end{array}\right]$ is the Jacobin transformation matrix, $\eta=\left[\eta_{x}, \eta_{y}, \eta_{\psi}\right]^{T} \in$ $R^{3}$ represents the Earth fixed positions and heading, respectively, $v=\left[v_{x}, v_{y}, v_{\psi}\right]^{T} \in R^{3}$ denotes the body fixed surge, sway, and yaw velocities, respectively, $d(v, \eta, t) \in R^{3}$ is the unknown disturbance, $M, C(v)$ and $D(v)$ are the inertia matrix, coriolis and centripetal terms, and the damping matrix respectively, $g(\eta)$ is an unknown vector of restoring forces due to buoyancy and gravitational forces and moments, $\tau$ is the generalized control vector produced jointly by all actuators. $\Phi(u)$ is the input nonlinearity which denotes input dead-zone and saturation. Next, assumptions and lemmas required for subsequent developments are presented.

Assumption 1: [15] The considered control input $\Phi(u)$ satisfies the saturation and dead-zone nonlinearity. That is, the control signal $u(t)=$ $\left[u_{1}(t), u_{2}(t), u_{3}(t)\right]^{T}$ is constrained by the saturation value $u_{\max }$ and dead-zone value $u_{0}$, expressed by

$$
\begin{array}{ll}
u_{i}(t)-u_{i 0} \leqslant u_{i \max }, & \text { for } u_{i} \geqslant u_{i 0}(t) \\
u_{i}(t)+u_{i 0} \geqslant-u_{i \max }, & \text { for } u_{i} \leqslant-u_{i 0}(t)(2)
\end{array}
$$

where, without loss of generality, a conservative common saturation limit $u_{i \max }$ and dead zone $u_{i 0}$ have been adopted to streamline the analysis. Equation (2) can be written as

$$
\begin{aligned}
& \Phi\left(u_{i}\right)=\chi\left(u_{i}-u_{i 0}\right)\left(u_{i}-u_{i 0}\right), \quad \text { for } u_{i} \geqslant u_{i 0}(t) \\
& \Phi\left(u_{i}\right)=\chi\left(u_{i}+u_{i 0}\right)\left(u_{i}+u_{i 0}\right), \quad \text { for } u_{i} \leqslant-u_{i 0}(t) \\
& \Phi\left(u_{i}\right)=0, \quad \text { for }-u_{i 0}(t) \leqslant u_{i} \leqslant u_{i 0}(t)
\end{aligned}
$$

where $i=1,2,3$ and

$$
\chi(.)= \begin{cases}\frac{u_{i \max }}{u_{i}-u_{i 0}}, \quad & \text { if } u_{i}-u_{i 0}>u_{i \max } \\
1, & \left\{\begin{array}{c}
\text { if } u_{i}-u_{i 0} \leqslant u_{i \max } \\
\text { for } u_{i} \geqslant u_{i 0} \\
\text { if } u_{i}+u_{i 0} \geqslant-u_{i \max } \\
\text { for } u_{i} \leqslant-u_{i 0}
\end{array}\right. \\
-\frac{u_{i \max }}{u_{i}+u_{i 0}}, & \text { if } u_{i}+u_{i 0}<-u_{i \max }\end{cases}
$$

such that $0<\chi() \leqslant$.1 .

Assumption 2: [11] For the continuous functions $d_{i}(v, \eta, t): R^{3} \times R^{3} \times R^{+} \rightarrow R, i=1,2,3$, there exists known smooth functions $\rho_{i}(v, \eta)$ and unknown bounded constants $\theta_{i}$ such that $\forall(v, \eta, t) \in$ $R^{3} \times R^{3} \times R^{+}$satsify

$$
\left|d_{i}(v, \eta, t)\right| \leqslant \rho_{i}(v, \eta) \theta_{i}
$$

Lemma 1: $[4,23]$ For bounded initial conditions, if there exists a $C^{1}$ continuous and the positive definite Lyapunov function $V(x)$ satisfying $\gamma_{1}(\|x\|) \leqslant V(x) \leqslant \gamma_{2}(\|x\|)$, such that $\dot{V}(x) \leqslant$ $-\kappa V(x)+c$, where $\gamma_{1}, \gamma_{2}: R^{n} \rightarrow R$ are class $K$ functions and $c$ is a positive constant, then the solution $x(t)$ is uniformly bounded.

Remark 1: Assumption 1 implies that the input nonlinearity $\Phi(u)$ of the underwater robot satisfies the conservative common saturation and deadzone. Thus, the control input $u(t)$ is constrained by saturation $u_{\max }$ and dead-zone value $u_{0}$. Assumption 2 means that the time-varying multivariable disturbance term $d_{i}(v, \eta, t)$ are bounded which generally includes exogenous effects and uncertainties. It is apparent that the boundary of disturbance term $d_{i}(v, \eta, t)$ are unknown due to the unknown $\theta_{i}$. Hence, Assumption 2 is reasonable. 
In this paper, the control objective is to follow a given desired trajectory of the underwater robot in the presence of parametric uncertainties, input nonlinearity and the disturbance form the external environment. For the desired trajectory $\eta_{d}$, the proposed robust adaptive tracking control must ensure that all closed-loop signals are bounded, and the tracking errors converge to a very small neighborhood of the origin, i.e. $\lim _{t \rightarrow \infty}\left\|y-\eta_{d}\right\|<\varepsilon_{t}$ for any $\varepsilon_{t}>0$.

\section{Adaptive Variable Structure Tracking Control}

We employ an approximation-based control in combination with VSC formulated through backstepping control of the system dynamics to follow a desired trajectory of the underwater robot (1). The full-state feedback tracking control will be developed for the system (1) with input nonlinearities. Rigorous stability analysis through Lyapunov method is carried out to show the semi-global uniform boundedness of the closed-loop system.

\subsection{Neural Network Approximators}

Neural networks (NNs) are often used as approximators for system parametric uncertainties due to their inherent approximation capabilities [28]. A class of linearly parameterized $\mathrm{NN}$ used to approximate the continuous function $\omega(Z): R^{q} \rightarrow R$ may be represented as

$$
\omega(Z)=\widehat{W}^{T} \phi(Z)+\varepsilon(Z)
$$

where $Z=\left[z_{1}, z_{2}, \ldots, z_{q}\right]^{T} \in R^{q}$ is the input vector of the NNs, $\widehat{W} \in R^{p}$ is a vector of adaptable weights, $\phi(Z)=\left[\phi_{1}, \phi_{2}, \ldots, \phi_{p}\right]^{T}$ is the vector of known continuous (linear or nonlinear) basis functions, and $\varepsilon$ is the approximation error, bounded over the compact set $\Omega_{Z}$, i.e., $|\varepsilon(Z)| \leqslant \bar{\varepsilon}_{0}, \forall Z \in \Omega_{Z}$, where $\bar{\varepsilon}_{0}>0$ is an unknown constant. A linearly parameterized neural network can smoothly approximate $\omega(Z)$ over the compact set $\Omega_{Z} \in R^{q}$ to arbitrary accuracy as

$$
\omega(Z)=W^{* T} \phi(Z)+\varepsilon^{*}(Z), \forall Z \in \Omega_{Z} \subset R^{q}
$$

where $W^{*}$ denotes the optimal weights, $\varepsilon^{*}(Z)$ is the approximation error for the case where $\widehat{W}=W^{*}$. Under the optimal weight value, there exists

$$
\left\|\omega(Z)-W^{* T} \phi(Z)\right\|=\left\|\varepsilon^{*}(Z)\right\| \leqslant\|\bar{\varepsilon}\|
$$

where the optimal weight value of $\mathrm{NN}$ is defined as

$$
W^{*}=\arg \min _{\widehat{W} \in \Omega_{\omega}}\left[\sup _{Z \in S_{Z}}|\widehat{\omega}(Z \mid \widehat{W})-\omega(Z)|\right]
$$

Here, $S_{Z} \subset R^{n}$ is an allowable set of the state vector, and $\Omega_{\omega}$ is a valid field of the parameter. We consider Gaussian Radial Basis Function Neural Network (RBFNN) [29] which is a particular network architecture that uses $l$ Gaussian functions of the form

$$
\phi_{i}(Z)=\exp \left[\frac{-\left(Z-\mu_{i}\right)^{T}\left(Z-\mu_{i}\right)}{\eta_{i}^{2}}\right], i=1,2, \ldots, l
$$

where $\mu_{i}=\left[\mu_{i 1}, \mu_{i 2}, \ldots, \mu_{i q}\right]^{T}$ is the center of the receptive field and $\eta_{i}$ is the width of the Gaussian function.

\subsection{Adaptive Tracking Control Design with Full-State Feedback}

In this subsection, we develop the tracking control scheme for the case where all states are available. Full state feedback tracking control is developed using adaptive NN, VSC and parameter adaptation in combination with backstepping control [30] of the system dynamics. To develop the robust adaptive tracking control, we define $\xi=J(\eta) v$ which is an auxiliary design variable, the error variables $z_{1}=\eta-\eta_{d}$ and $z_{2}=\xi-\alpha_{1}$. It is apparent that $v \rightarrow 0$ if $\xi \rightarrow 0$ due to the nonsingularity of $J(\eta)$.

Step 1: Considering (1) and differentiating $z_{1}$ with respect to time yields

$$
\dot{z}_{1}=\dot{\eta}-\dot{\eta}_{d}=\xi-\dot{\eta}_{d}=z_{2}+\alpha_{1}-\dot{\eta}_{d}
$$

The virtual control law $\alpha_{1}$ is designed as

$$
\alpha_{1}=-K_{1} z_{1}+\dot{\eta}_{d}
$$

where $K_{1}=K_{1}^{T}>0$.

Substituting (12) into (11), we obtain

$$
\dot{z}_{1}=-K_{1} z_{1}+z_{2}
$$


Consider the Lyapunov function candidate $V_{1}=$ $\frac{1}{2} z_{1}^{T} z_{1}$. The time derivative of $V_{1}$ is given by

$$
\dot{V}_{1}=-z_{1}^{T} K_{1} z_{1}+z_{1}^{T} z_{2}
$$

The first term on the right-hand side is negative, and the second term will be canceled in the next step.

Step 2: Considering (1) and differentiating $z_{2}$ with respect to time, we have

$$
\begin{aligned}
\dot{z}_{2} & =\dot{\xi}-\dot{\alpha}_{1}=\dot{J}(\eta) v+J(\eta) \dot{v}-\dot{\alpha}_{1} \\
& =\dot{J}(\eta) v+J(\eta) M^{-1}(-C(v) v-D(v) v \\
& -g(\eta)+\Phi(u)+d(v, \eta, t))-\dot{\alpha}_{1}
\end{aligned}
$$

where $\dot{\alpha}_{1}=\ddot{\eta}_{d}-K_{1} \dot{z}_{1}$.

To tackle the dead-zone of the input for the underwater robot, the VSC is employed to design the robust tracking control in this paper. The sliding surface is chosen as

$$
S=c_{1} z_{1}+z_{2}
$$

where $c_{1}>0$.

Consider the following Lyapunov function candidate

$$
V_{2}^{*}=V_{1}+\frac{1}{2 \delta} S^{T} M S
$$

where $\delta>0$ is a design parameter which is an unknown auxiliary constant and is defined as follows

$$
0<\underline{\delta} \leqslant \delta \leqslant \bar{\delta} \leqslant \chi_{i}(.) \leqslant 1
$$

where $\bar{\delta} \geqslant \underline{\delta}>0$.

Differentiating $V_{2}^{*}$, we have

$$
\dot{V}_{2}^{*} \leqslant-z_{1}^{T} K_{1} z_{1}+z_{1}^{T} z_{2}+\frac{1}{\delta} S^{T} M \dot{S}
$$

Invoking (16), we have

$$
z_{2}=S-c_{1} z_{1}
$$

Considering (14), (15), and Assumption 2, substituting (20) into (19) yields

$$
\begin{aligned}
\dot{V}_{2}^{*} & \leqslant-z_{1}^{T} K_{1} z_{1}-c_{1} z_{1}^{T} z_{1}+\frac{1}{\delta} S^{T}\left(\delta z_{1}-c_{1} M K_{1} z_{1}\right. \\
& +c_{1} M z_{2}+M \dot{J}(\eta) v-J(\eta) C(v) v \\
& \left.-J(\eta) D(v) v-J(\eta) g(\eta)-M \dot{\alpha}_{1}\right) \\
& +\frac{S^{T} J(\eta)}{\delta} \Phi(u)+\lambda S^{T} \operatorname{Sgn}(S) \rho(v, \eta) \theta
\end{aligned}
$$

where $\lambda=\|J(\eta)\|, \quad \operatorname{Sgn}(S):=\operatorname{diag}\left\{\operatorname{sgn}\left(S_{j}\right)\right\}$, $\rho(v, \eta)=\operatorname{diag}\left\{\rho_{j}(v, \eta)\right\}$ and $\theta=\left[\theta_{1} / \delta, \theta_{2} / \delta, \theta_{3} / \delta\right]^{T}$, $j=1,2,3$.

Since $M, C(v), D(v), \delta$ and $\theta$ are all unknown, the robust tracking control cannot be directly designed. To solve this problem, we use adaptation technique to handle the unknown parameter $\theta$ and $\mathrm{NN}$ is employed to approximate the unknown term $h(Z)$ which is given by

$$
\begin{aligned}
h(Z) & =-\frac{1}{\delta}\left(\delta z_{1}-c_{1} M K_{1} z_{1}+c_{1} M z_{2}+M \dot{J}(\eta) v\right. \\
& -J(\eta)(C(v) v+D(v) v+g(\eta))-M \dot{\alpha}(2) 2)
\end{aligned}
$$

where $Z=\left[\eta^{T}, v^{T}, \alpha_{1}^{T}, \dot{\alpha}_{1}^{T}\right]$.

The approximation output of $\mathrm{NN}$ for the unknown term $h(Z)$ can be expressed as

$$
\widehat{h}(Z)=\widehat{W}^{T} \phi(Z)
$$

where $\widehat{W}=\operatorname{blockdiag}\left[\widehat{W}_{1}^{T}, \widehat{W}_{2}^{T}, \widehat{W}_{3}^{T}\right]^{T}$ are the neural network parameters, $\phi(Z)=\left[\phi_{1}^{T}(Z), \phi_{2}^{T}(Z)\right.$, $\left.\phi_{3}^{T}(Z)\right]^{T}$ represents the basis functions, and $\widehat{W}^{T} \phi(Z)$ approximates $W^{* T} \phi(Z)$ given by

$$
\rho(Z)=W^{* T} \phi(Z)+\varepsilon
$$

Substituting (24) into (21), we obtain

$$
\begin{aligned}
\dot{V}_{2}^{*} & \leqslant-z_{1}^{T} K_{1} z_{1}-c_{1} z_{1}^{T} z_{1} \\
& -S^{T} W^{* T} \phi(Z)+\lambda S^{T} \operatorname{Sgn}(S) \rho(v, \eta) \theta \\
& -S^{T} \varepsilon+S^{T} \frac{J(\eta)}{\delta} \Phi(u)
\end{aligned}
$$

Considering the nonsingular matrix $J(\eta)$, define $\vartheta=J(\eta)^{T} S=\left[\vartheta_{1}, \vartheta_{2}, \vartheta_{3}\right]^{T}$. Then, the following VSC law with nonlinearity/dead-zone is proposed:

$$
u_{i}= \begin{cases}-\bar{u}_{i 0}+u_{i 0}, & \text { if } \vartheta_{i}<0 \\ 0, & \text { if } \vartheta_{i}=0 \\ -\bar{u}_{i 0}-u_{i 0}, & \text { if } \vartheta_{i}>0\end{cases}
$$

where $\quad \bar{u}_{i 0}=\left(S^{T} \Lambda S+\left\|S^{T}\right\|\left\|\widehat{W}^{T} \phi(Z)\right\|+\right.$ $\left.\lambda\left\|S^{T}\right\|\|\operatorname{Sgn}(S) \rho(v, \eta) \widehat{\theta}\|\right) \frac{\vartheta_{i}}{\|\vartheta\|^{2}}, \Lambda=\Lambda^{T}>0$ is an 
appropriate dimension positive definite matrix, and $u_{i 0}, i=1,2,3$ is the known dead-zone values.

To further design and analyze, the following Lemma is required.

Lemma 2: If the input nonlinearities $\Phi(u)$ satisfying Assumptions 1, the VSC law (26) can make the following inequality always hold:

$$
\begin{aligned}
S^{T} J(\eta) \Phi(u) & \leqslant-\delta\left(S^{T} \Lambda S+\left\|S^{T}\right\|\left\|\widehat{W}^{T} \phi(Z)\right\|\right. \\
& \left.+\lambda\left\|S^{T}\right\|\|\operatorname{Sgn}(S) \rho(v, \eta) \widehat{\theta}\|\right)(27)
\end{aligned}
$$

Proof: From equations (3) and (26), $u_{i}>u_{i 0}$ implies that $\vartheta_{i}<0$ and thus

$$
\left(u_{i}-u_{i 0}\right) \Phi\left(u_{i}\right)=-\bar{u}_{i 0} \Phi\left(u_{i}\right) \geqslant \delta\left(u_{i}-u_{i 0}\right)^{2}=\bar{u}_{i 0}^{2}
$$

Whereas for $u_{i}<-u_{i 0}, \vartheta_{i}>0$ and there yields

$\left(u_{i}+u_{i 0}\right) \Phi\left(u_{i}\right)=-\bar{u}_{i 0} \Phi\left(u_{i}\right) \geqslant \delta\left(u_{i}+u_{i 0}\right)^{2}=\bar{u}_{i 0}^{2}$

Considering (28) and (29) yields

$$
\begin{aligned}
\vartheta_{i} \Phi\left(u_{i}\right) & \leqslant-\delta \frac{\vartheta_{i}^{2}}{\|\vartheta\|^{2}}\left(S^{T} \Lambda S+\left\|S^{T}\right\|\left\|\widehat{W}^{T} \phi(Z)\right\|\right. \\
& \left.+\lambda\left\|S^{T}\right\|\|\operatorname{Sgn}(S) \rho(v, \eta) \widehat{\theta}\|\right)
\end{aligned}
$$

Therefore, the following inequality always holds:

$$
\begin{aligned}
S^{T} J(\eta) \Phi(u) & =\sum_{i=1}^{3} \vartheta_{i} \Phi\left(u_{i}\right) \\
& \leqslant-\delta\left(S^{T} \Lambda S+\left\|S^{T}\right\|\left\|\widehat{W}^{T} \phi(Z)\right\|\right. \\
& \left.+\lambda\left\|S^{T}\right\|\|\operatorname{Sgn}(S) \rho(v, \eta) \widehat{\theta}\|\right)(31)
\end{aligned}
$$

This concludes the proof, and the lemma shows that the input nonlinearity combining with the sliding surface satisfies a given inequation (31). $\diamond$

Substituting (31) into (25) yields

$$
\begin{aligned}
\dot{V}_{2}^{*} & \leqslant-z_{1}^{T} K_{1} z_{1}-c_{1} z_{1}^{T} z_{1}-S^{T} \Lambda S-S^{T} W^{* T} \phi(Z) \\
& +S^{T} \widehat{W}^{T} \phi(Z)-S^{T} \varepsilon-\left\|S^{T}\right\|\left\|\widehat{W}^{T} \phi(Z)\right\| \\
& -S^{T} \widehat{W}^{T} \phi(Z)+\lambda S^{T} \operatorname{Sgn}(S) \rho(v, \eta) \theta \\
& -\lambda\left\|S^{T}\right\|\|\operatorname{Sgn}(S) \rho(v, \eta) \widehat{\theta}\| \\
& +\lambda S^{T} \operatorname{Sgn}(S) \rho(v, \eta) \widehat{\theta}-\lambda S^{T} \operatorname{Sgn}(S) \rho(v, \eta) \widehat{\theta} \\
& \leqslant-z_{1}^{T} K_{1} z_{1}-c_{1} z_{1}^{T} z_{1}-S^{T} \Lambda S-S^{T} W^{* T} \phi(Z) \\
& +S^{T} \widehat{W}^{T} \phi(Z)-S^{T} \varepsilon+\lambda S^{T} \operatorname{Sgn}(S) \rho(v, \eta) \widehat{\theta} \\
& -\lambda S^{T} \operatorname{Sgn}(S) \rho(v, \eta) \theta
\end{aligned}
$$

To analyze the stability of error signals $\widetilde{W}_{i}:=$ $\widehat{W}_{i}-W_{i}^{*}$ and $\widetilde{\theta}:=\widehat{\theta}-\theta^{*}$, the augmented Lyapunov function can be written as

$$
V_{2}=V_{2}^{*}+\frac{1}{2} \sum_{i=1}^{3} \widetilde{W}_{i}^{T} \Lambda_{i}^{-1} \widetilde{W}_{i}+\frac{1}{2} \widetilde{\theta}^{T} \Lambda_{0}^{-1} \widetilde{\theta}
$$

where $\Lambda_{i}=\Lambda_{i}^{T}>0, i=0,1,2,3$.

Differentiating (33) along (32) yields

$$
\begin{aligned}
\dot{V}_{2} & \leqslant-z_{1}^{T} K_{1} z_{1}-c_{1} z_{1}^{T} z_{1}-S^{T} \Lambda S-S^{T} W^{* T} \phi(Z) \\
& +S^{T} \widehat{W}^{T} \phi(Z)-S^{T} \varepsilon+\sum_{i=1}^{3} \widetilde{W}_{i}^{T} \Lambda_{i}^{-1} \dot{\hat{W}}_{i} \\
& +\widetilde{\theta}^{T} \Lambda_{0}^{-1} \dot{\widehat{\theta}}-\lambda S^{T} \operatorname{Sgn}(S) \rho(v, \eta) \theta \\
& +\lambda S^{T} \operatorname{Sgn}(S) \rho(v, \eta) \hat{\theta}
\end{aligned}
$$

Considering $-S^{T} \varepsilon \leqslant \frac{1}{2}\left(S^{T} S+\varepsilon^{T} \varepsilon\right)$, inequality (34) can be expressed as

$$
\begin{aligned}
\dot{V}_{2} & \leqslant-z_{1}^{T} K_{1} z_{1}-c_{1} z_{1}^{T} z_{1}-S^{T} \Gamma S+\sum_{i=1}^{3} \widetilde{W}_{i}^{T} \phi_{i}(Z) S_{i} \\
& +\sum_{i=1}^{3} \widetilde{W}_{i}^{T} \Lambda_{i}^{-1} \dot{\widehat{W}}_{i}+\frac{1}{2}\|\varepsilon\|^{2} \\
& +\lambda S^{T} \operatorname{Sgn}(S) \rho(v, \eta) \widetilde{\theta}+\widetilde{\theta}^{T} \Lambda_{0}^{-1} \dot{\hat{\theta}}
\end{aligned}
$$

where $\Gamma=\Lambda-\frac{1}{2} I_{3 \times 3}$.

Consider the adaptation laws for $\widehat{W}_{i}$ and $\widehat{\theta}$ as

$$
\begin{aligned}
\dot{\widehat{W}}_{i} & =-\Lambda_{i}\left(\phi_{i}(Z) S_{i}+\beta_{i} \widehat{W}_{i}\right) \\
\dot{\hat{\theta}} & =-\Lambda_{0}\left(\operatorname{Sgn}(S) \rho(v, \eta) S+\beta_{0} \widehat{\theta}\right)
\end{aligned}
$$

where $\beta_{i}>0$ and $\beta_{0}>0$.

Substituting (36) and (37) into (35) yields

$$
\begin{aligned}
\dot{V}_{2} & \leqslant-z_{1}^{T} K_{1} z_{1}-c_{1} z_{1}^{T} z_{1}-S^{T} \Gamma S-\sum_{i=1}^{3} \frac{\beta_{i}}{2}\left\|\widetilde{W}_{i}\right\|^{2} \\
& +\sum_{i=1}^{3} \frac{\beta_{i}}{2}\left\|W_{i}^{*}\right\|^{2}-\frac{\beta_{0}}{2}\|\widetilde{\boldsymbol{\theta}}\|^{2}+\frac{\beta_{0}}{2}\left\|\theta^{*}\right\|^{2}+\frac{1}{2}\|\varepsilon\|^{2} \\
& \leqslant-\kappa V_{2}+C
\end{aligned}
$$


where

$$
\begin{aligned}
& \kappa:=\min \left(\begin{array}{c}
2 \lambda_{\min }\left(K_{1}+c_{1} I_{3 \times 3}\right), \frac{2 \delta \lambda_{\min }(\Gamma)}{\lambda_{\max }(M)}, \\
\min _{i=1,2,3}\left(\frac{\beta_{i}}{\lambda_{\max }\left(\Lambda_{i}^{-1}\right)}\right), \frac{\beta_{0}}{\lambda_{\max }\left(\Lambda_{0}^{-1}\right)}
\end{array}\right) \\
& C:=\sum_{i=1}^{3} \frac{\beta_{i}}{2}\left\|W_{i}^{*}\right\|^{2}+\frac{\beta_{0}}{2}\left\|\theta^{*}\right\|^{2}+\frac{1}{2}\|\bar{\varepsilon}\|^{2}
\end{aligned}
$$

To ensure that $\kappa>0$, the matrices $K_{1}, \Lambda$ and the design parameter $c_{1}$ are chosen to satisfy the following conditions:

$$
\lambda_{\min }\left(K_{1}+c_{1} I_{3 \times 3}\right)>0, \quad \lambda_{\min }(\Gamma)>0
$$

The above design procedure can be summarized in the following theorem, which contains the results for full state feedback control of the system dynamics (1) with the input dead-zone and saturation.

Theorem 1: Consider the underwater robot dynamics (1) with the Assumption 1 and Assumption 2, given that the system initial conditions are bounded, and that full state information is available, under the control law (26) and adaptation laws (36) and (37), the closed-loop system is semi-globally uniformly stable and error signals $z_{1}, S, \widetilde{W}$ and $\widetilde{\theta}$ remains within the compact sets $\Omega_{z 1}, \Omega_{z 2}$ and $\Omega_{\theta}$ respectively, defined by

$$
\begin{aligned}
\Omega_{z_{1}} & :=\left\{z_{1} \in R^{3} \mid\left\|z_{1}\right\| \leqslant \sqrt{D}\right\} \\
\Omega_{S} & :=\left\{z_{2} \in R^{3} \mid\left\|z_{2}\right\| \leqslant \sqrt{\frac{D}{\lambda_{\max }\left(\Gamma^{-1}\right)}}\right\} \\
\Omega_{\widetilde{W}} & :=\left\{\widetilde{W} \in R^{l} \mid\|\widetilde{W}\| \leqslant \sqrt{\frac{D}{\lambda_{\max }\left(\Lambda_{i}^{-1}\right)}}\right\} \\
\Omega_{\widetilde{\theta}} & :=\left\{\widetilde{\theta} \in R^{3} \mid\|\widetilde{\boldsymbol{\theta}}\| \leqslant \sqrt{\frac{D}{\lambda_{\max }\left(\Lambda_{0}^{-1}\right)}}\right\}
\end{aligned}
$$

where $D=2\left(V_{2}(0)+\frac{C}{\kappa}\right)$ with $C$ and $\kappa$ as defined in (39).

Proof: Utilizing (38) and Lemma 2, it can directly show that the signals $z_{1}, S, \widetilde{W}$ and $\widetilde{\theta}$ are semiglobally uniformly bounded. For completeness, the details of the proof are provided here. Multiplying (38) by $e^{K t}$ and performing integration on the inequality yields

$$
V_{2} \leqslant\left(\left.V_{2}\right|_{t=0}-\frac{C}{\kappa}\right) e^{-\kappa t}+\frac{C}{\kappa} \leqslant\left. V_{2}\right|_{t=0}+\frac{C}{\kappa}
$$

Considering (33), the bounds of $z_{1}$ can be found as

$$
\frac{1}{2}\left\|z_{1}\right\|^{2} \leqslant\left. V_{2}\right|_{t=0}+\frac{C}{\kappa}
$$

Hence, there yields

$$
\left\|z_{1}\right\| \leqslant \sqrt{2\left(\left.V_{2}\right|_{t=0}+\frac{C}{\kappa}\right)}
$$

and the bounds of $S, \widetilde{W}$ and $\widetilde{\theta}$ can be similarly shown. This concludes the proof. $\diamond$

Remark 2: The uniform boundedness of all closed-loop signals established in Theorem 1 is semi-global due to the use of approximation-based control, which are only valid within a compact set. In the design process of tracking control, the auxiliary design constant $\delta$ is used which satisfies $0<$ $\delta \leqslant \chi_{i}() \leqslant$.1 . We treat it as an unknown part of the compound system uncertainty $h(Z)$ and the unknown parameter $\theta$. Then, $h(Z)$ is approximated by the NN and $\theta$ is canceled using parameter adaptation, respectively. $\delta$ does not appear in the design of parameter adaptive laws and control law.

\section{Simulation Results}

In our simulation study, extensive simulation has been carried to demonstrate the effectiveness of the state feedback tracking control designed in Setion 3. Assume that the underwater robot is a scale replica of a underwater robot which can be represented using (1) and detailed parameters obtained from [27,31]. As detailed in [27], the parameters of the dynamical model of Cybership II are as follows:

$$
\begin{aligned}
M= & {\left[\begin{array}{ccc}
25.8 & 0 & 0 \\
0 & 33.8 & 1.09 \\
0 & 1.09 & 2.76
\end{array}\right], } \\
J(\eta)= & {\left[\begin{array}{ccc}
\cos \left(\eta_{\psi}\right) & -\sin \left(\eta_{\psi}\right) & 0 \\
\sin \left(\eta_{\psi}\right) & \cos \left(\eta_{\psi}\right) & 0 \\
0 & 0 & 1
\end{array}\right], } \\
C(v)= & {\left[\begin{array}{ccc}
0 & 0 & C_{13} \\
0 & 0 & -25.8 v_{x} \\
C_{31} & 25.8 u & 0
\end{array}\right], } \\
D(v)= & {\left[\begin{array}{ccc}
D_{11} & 0 & 0 \\
0 & D_{22} & D_{23} \\
0 & D_{32} & D_{33}
\end{array}\right] . }
\end{aligned}
$$


where $C_{31}=-C_{13}=33.8 v_{y}+1.09 v_{\psi}, \quad D_{11}=$ $0.723+1.33\left|v_{x}\right|+57.8 v_{x}^{2}, D_{22}=0.861+36.3\left|v_{y}\right|+$ $0.01\left|v_{\psi}\right|, \quad D_{23}=-0.108+0.005\left|v_{y}\right|+0.01\left|v_{\psi}\right|$, $D_{32}=-0.105-5.04\left|v_{y}\right|+0.001\left|v_{\psi}\right|$ and $D_{33}=$ $0.5+0.0007\left|v_{y}\right|+0.001\left|v_{\psi}\right|$.

The disturbance $d$ is defined as a stochastic, time varying force dependent on $\eta$ and $t$. Its expression is [4]

$$
d(\eta, t)=J(\eta)^{T} f_{d}(t)
$$

where $f_{d}=[3+0.7 \sin (0.4 t)+0.2 \cos (0.2 t)+$ $0.2 \sin (0.3 t), 0,0]^{T}$.

The control objective is to follow a certain desired trajectory of the underwater robot. Here, we suppose that the expect trajectory is $\eta_{d}=$ $[10 \sin (0.05 t), 0,0]^{T}$. In this simulation study, the RBFNNs are used as feedforward approximators of function uncertainties $h(Z)$. Using (12), $\alpha_{1}$ can be computed. According to (26), (36) and (37), the control law and parameter updated laws are proposed.

By choosing $K_{1}=\operatorname{diag}\{0.5\}_{3 \times 3}, \Lambda_{1}=\Lambda_{2}=$ $\Lambda_{3}=\operatorname{diag}\left\{1 \times 10^{-6}\right\}, \beta_{i}=1 \times 10^{-5}, \varepsilon=0.01, c_{1}=$ 0.8 , the dead-zone values $u_{10}=u_{20}=0.5, u_{30}=$ 0.2 , and the saturation values $u_{1 \max }=u_{2 \max }=5.0$, $u_{3 \max }=3.5 . \widehat{W}=\operatorname{diag}\left\{\widehat{\mathrm{W}}_{1}^{\mathrm{T}}, \widehat{\mathrm{W}}_{2}^{\mathrm{T}}, \widehat{\mathrm{W}}_{3}^{\mathrm{T}}\right\}$ is the adaptable parameter of RBFNN. It is easy to check that the condition (40) are satisfied. The initial state conditions are arbitrarily chosen as $\eta_{0}=[0.5,2.0,0.1]^{T}$, $v_{0}=[0.2,0,0]^{T}, \widehat{W}_{i}=[0,0,0]^{T}, i=1,2,3$, and $\widehat{\theta}=$ $[0,0,0]^{T}$.

Under the proposed tracking control, from figures 1 and 2, it can be observed that the tracking performance is satisfactory under the time-varying disturbance and parametric uncertainty that act on it. According to Figures 3, we can see that the surge, sway and yaw velocities response of underwater robots can also quickly converge to desired values. The input saturation can be observed form the Figure 4 which is produced by the input constraint. From Figure 5, the convergence performance of neural network weight value norms can be guaranteed with the proposed tracking control scheme. Based on these simulation results, we can obtain that the proposed full state feedback tracking control is valid for the underwater robot in presence of parametric uncertainties, external disturbance, input dead-zone and input saturation.
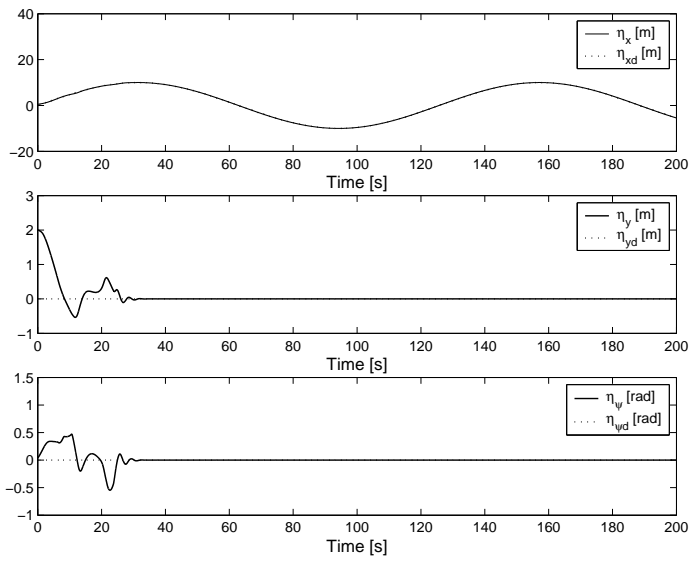

Fig. 1. Positions and heading response of underwater robots under the proposed control.

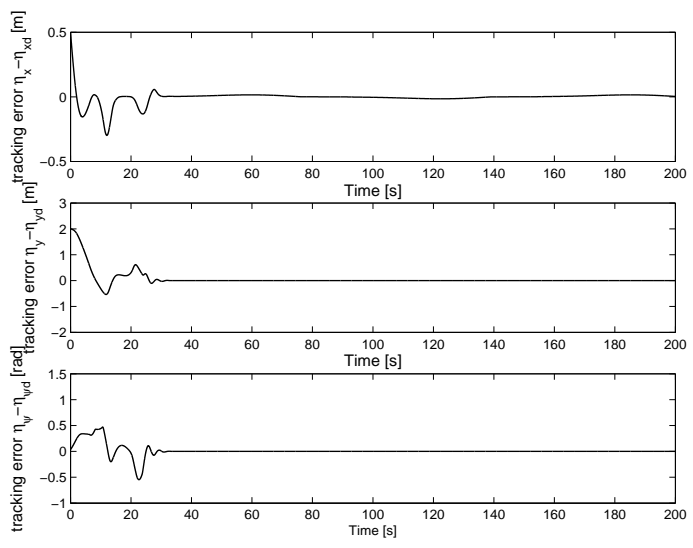

Fig. 2. Tacking errors.
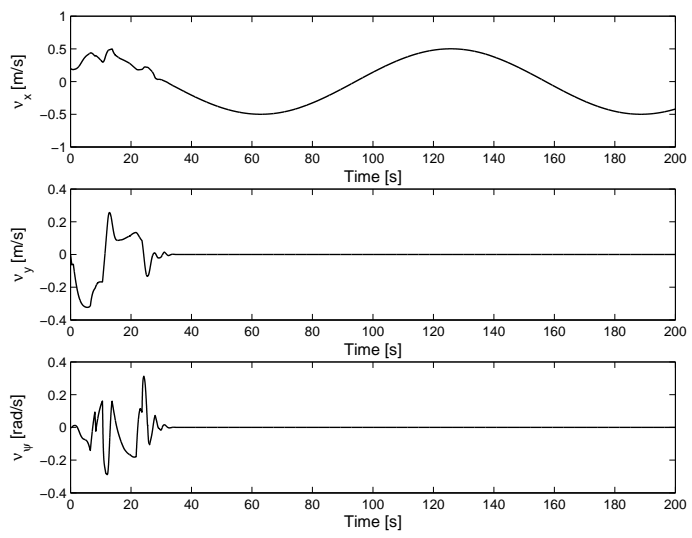

Fig. 3. Surge, sway and yaw velocities response of underwater robots. 

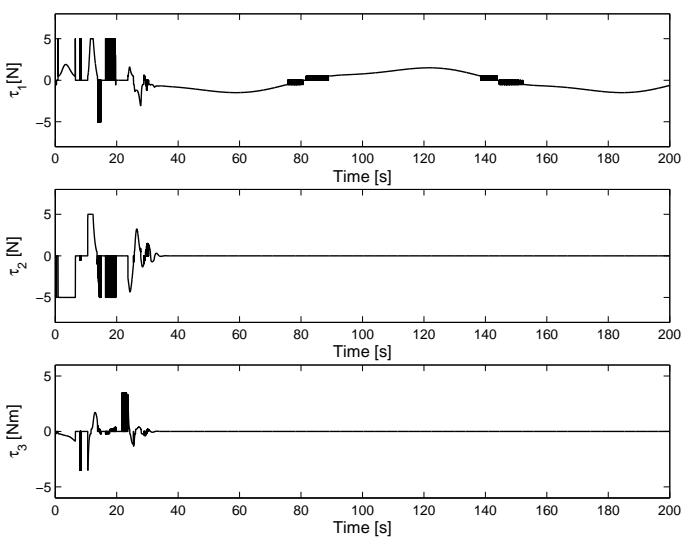

Fig. 4. Control input of underwater robots.
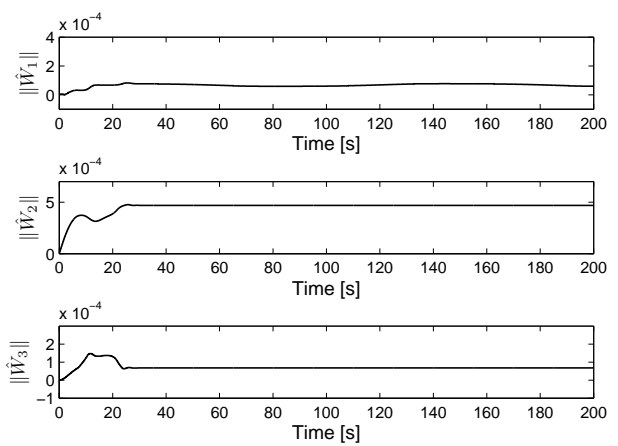

Fig. 5. Norms of the Neural network weight values.

\section{Conclusions}

Robust adaptive tracking control has been proposed for the underwater robot with parametric uncertainties, external disturbance, input dead-zone and saturation in this paper. To tackle saturation and deadzone nonlinearities of actuators, the sliding mode tracking control using the full state feedback has been proposed for the underwater robot with backstepping method and neural networks. In this paper, two auxiliary design variables have been introduced to design backstepping control to simplify the derivation computation of the virtual control law and to tackle the input nonlinearity. Finally, simulation studies have been given to illustrate the effectiveness of the proposed tracking control.

\section{Acknowledgments}

This work is partially supported by the Jiangsu Natural Science Foundation of China under grant SBK2008390. The authors also gratefully acknowledge the helpful comments and suggestions of the reviewers, which have improved the presentation.

\section{References}

1. P. Kiriazov, "Robust feedback stabilization of underwater robotic vehicles," Robotics and Autonomous Systems, 21, 415-423(1997).

2. T. Chatchanayuenyong and M. Parnichkun, "Neural network based-time optimal sliding mode control for an autonomous underwater robot," Mechatronics, 16, 471-478(2006).

3. M. Lee and H. S. Choi, "A robust neural controller for underwater robot manipulators," IEEE Transactions on Neural Networks, 11, 1465-1470(2000).

4. K. P. Tee and S. S. Ge, "Control of fully actuated ocean surface vessels using a class of feedforward approximators," IEEE Transactions on Control Systems Technology, 14, 750-756( 2006).

5. K. Ishii, T. Fujii, and T. Ura, "Neural network system for online controller adaptation and its application to underwater robot," IEEE International Conference on Robotics and Automation, 1998. 1, 756-761(1998).

6. M. Corradini and G. Orlando, "Robust stabilization of nonlinear uncertain plants with backlash or dead zone in the actuator," IEEE Transactions on Control Systems Technology, 10, 158-166(2002).

7. M. Corradini and G. Orlando, "Robust practical stabilization of nonlinear uncertain plants with input and output nonsmooth nonlinearities," IEEE Transactions on Control Systems Technology, 11, 196-203(2003).

8. G. Tao and P. V. Kokotovi ć, "Discrete-time adaptive control of plants with unknown output dead-zones," Automatica, 31, 287-291(1995).

9. J. Zhou, C. Wen, and Y. Zhang, "Adaptive output control of nonlinear systems with uncertain dead-zone nonlinearity," IEEE Transactions on Automatic Control, 51, 504-510(2006).

10. T. P. Zhang and S. S. Ge, "Adaptive neural control of mimo nonlinear state time-varying delay systems with unknown dead-zones and gain signs," Automatica, $\mathbf{4 3}$ 1021-1033(2007).

11. T. P. Zhang and S. S. Ge, "Adaptive dynamic surface control of nonlinear systems with unknown dead 
zone in pure feedback form," Automatica, 44, 18951903(2008).

12. J. R. Azinheira and A. Moutinho, "Hover control of an UAV with backstepping design including input saturations," IEEE Transactions on Control Systems Technology, 16, 517-526(2008).

13. Y. Y. Cao and Z. L. Lin, "Robust stability analysis and fuzzy-scheduling control for nonlinear systems subject to actuator saturation," IEEE Transactions on Fuzzy Systems, 11, 57-67(2003).

14. Y. S. Zhong, "Globally stable adaptive system design for minimum phase SISO plants with input saturation," Automatica, 41, 1539-1547(2005).

15. Q. L. Hu, G. F. Ma, and L. H. Xie, "Robust and adaptive variable structure output feedback control of uncertain systems with input nonlinearity," Automatica, 44, 552-559(2008).

16. J. Zhou, C. Y. Wen, and Y. Zhang, "Adaptive backstepping control of a class of uncertain nonlinear systems with unknown backlash-like hysteresis," IEEE transactions on Automatic Control, 49, 1751-1757(2004).

17. M. Chen, C. S. Jiang, and Q. X.Wu, "Robust adaptive control of uncertain time delay systems with FLS," International Journal of Innovative Computing, Information and Control, 4, 1551-1561(2008).

18. T. Zhang, S. S. Ge, and C. C. Hang, "Adaptive neural network control for strict-feedback nonlinear systems using backstepping design," Automatica, 36, 18351846(2000).

19. S. S. Ge, C. C. Hang, and T. Zhang, "Adaptive neural network control of nonlinear systems by state and output feedback," IEEE Transactions on System, Man, and Cybernetics - Part B: Cybernetics, 29, 818828(1999).

20. S. S. Ge, C. C. Hang, and T. Zhang, "Stable adaptive control for nonlinear multivariable systems with a triangular control structure," IEEE Transactions on Automatic Control, 45, 1211-1225(2000).

21. S. S. Ge and C. Wang, "Adaptive neural control of uncertain MIMO nonlinear systems," IEEE Transactions on Neural Networks, 15, 674-692(2004).

22. K. P. Tee, S. S. Ge, and F. E. H. Tay, "Adaptive neural network control for helicopters in vertical fight," IEEE Transactions on Control Systems Technology, 16, 753-762(2008).

23. S. S. Ge and C. Wang, "Direct adaptive NN control of a class of nonlinear systems," IEEE Transactions on Neural Networks, 13, 214-221(2002).

24. Y. P. Zhang, P. Y. Peng, and Z. P. Jiang, "Stable neural controller design for unknown nonlinear systems using backstepping," IEEE Transaction on Neural Networks, 11, 1347-1360(2000).

25. M. Chen, C. S. Jiang, and Q. X. Wu, "Backstepping control for a class of uncertain nonlinear systems with neural network," International Journal of Nonlinear Science, 3, 137-143(2007).

26. S. Sentürk, "Fuzzy regression control chart based on Alfa-cut approximation," International Journal of Computational Intelligence Systems, 3, 123140(2010).

27. T. I. Fossen, “Marine Control systems”, NJ: Trondheim, Norway: 2rd. Tapir Trykkeri, 2002.

28. S. S. Ge, C. C. Hang, T. H. Lee, and T. Zhang, "Stable adaptive neural network control", Norwell, USA: Kluwer Academic, 2001.

29. D. Marcek, M. Marcek, and J. Babel, "Granular RBF NN approach and statistical methods applied to modelling and forecasting high frequency data," International Journal of Computational Intelligence Systems, 2, 353-364(2009).

30. M. Chen, S. S. Ge, and Y. S. Choo, "Neural network tracking control of ocean surface vessels with input saturation," Proceedings of the IEEE International Conference on Automation and Logistics, 8589(2009).

31. O. M. Aamo and T. I. Fossen, "Controlling line tension in thruster assisted mooring systems," Proceedings of the 1999 IEEE International Conference on Control Applications, 2, 1104-1109(1999). 